\title{
Active Temperature compensation for MEMS capacitive sensor
}

\author{
Cuong Do, Member, IEEE, Ashwin A. Seshia, Fellow, IEEE
}

\begin{abstract}
Temperature variations are one of the most crucial factors that need to be compensated for in MEMS sensors. Many traditional methodologies require an additional circuit to compensate for temperature. This work describes a new active temperature compensation method for MEMS capacitive strain sensors without additional circuits. The proposed method is based on a complement 2-D capacitive structure design. It consumes zero-power, which is essential toward the realization of a lowpower temperature-compensated sensor in battery-powered or energy-
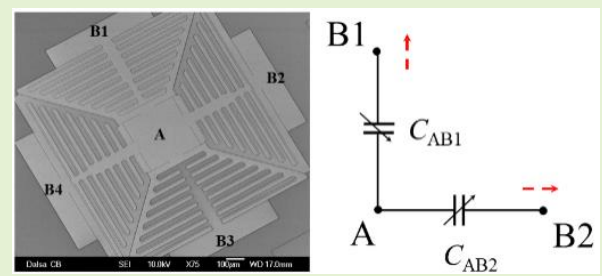
harvesting applications. The gauge factor of the developed MEMS capacitive strain sensor is 7 . The best result showing a capacitance variation of $1 \mathrm{ppm} /{ }^{\circ} \mathrm{C}$ compared with $13 \mathrm{ppm} /{ }^{\circ} \mathrm{C}$ on conventional design.
\end{abstract}

Index Terms-Active temperature compensation, Complement 2-D capacitive structure, Strain gauge.

\section{Introduction}

CarA APACITORs typically consist of two electrodes separated by an insulator. For parallel plate capacitors, the capacitance can be expressed as:

$$
C=\left(A \varepsilon_{0} \varepsilon_{r}\right) / d
$$

where $C$ is the capacitance, $A$ is the overlap area of the two plates, $\varepsilon_{0}$ is the permittivity of free space, $\varepsilon_{\mathrm{r}}$ is the relative permittivity of the dielectric material, and $d$ is the distance between the plates. Capacitive sensors are realized by varying any of the three parameters of a capacitor: the distance between two plates, the overlap area of plates, and the dielectric constant of an insulator.

Based on those physical changes, a wide variety of capacitive sensors have been developed, ranging from proximity sensing [1-5], position sensing [6, 7], humidity sensing [8,9], tilt sensing $[10,11]$, strain sensing $[12,13,14]$, etc. As the capacitance is not dependent on the properties of the plate material, and thermal expansion is generally isotropic in three dimensions, capacitive sensors are generally considered to having low-temperature dependence compared with other sensor methods, such as resistive based [15] or resonant based [16]. However, in sensing applications where the capacitive sensor adheres to package or other host materials, such as strain gauge as demonstrated in Fig. 1, the coefficient of thermal capacitance (CTC) will be affected and proportional to the

Manuscript received ....

Cuong Do was with the Engineering Department at the University of Cambridge. He is now with the College of Engineering and Computer Science, VinUniversity, Hanoi, Vietnam (email: cuong.dd@vinuni.edu.vn)

Ashwin A. Seshia is with the Engineering Department at the University of Cambridge, Cambridge, UK (email: aas41@eng.cam.ac.uk)

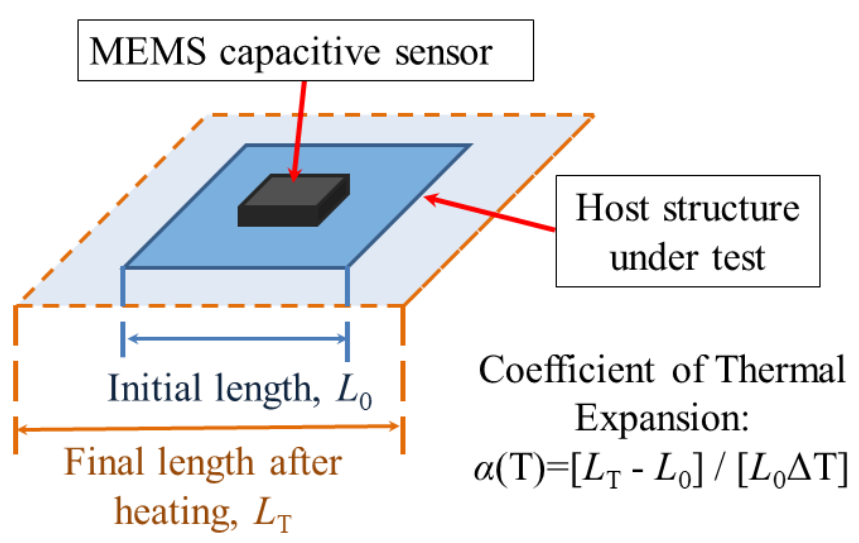

Fig. 1. Thermal expansion of host structure under test

complex combination of the coefficient of thermal expansion (CTE) of the adhesive and host materials.

Most materials expand and contract when the temperature changes. Every material has its own coefficient of thermal expansion (CTE), the change in length with temperature for a solid material can be expressed as

$$
\alpha(\mathrm{T})=\left[\boldsymbol{L}_{\mathrm{T}}-\boldsymbol{L}_{\mathbf{0}}\right] /\left[\boldsymbol{L}_{\mathbf{0}} \Delta \mathrm{T}\right]
$$

where $L_{0}$ and $L_{\mathrm{T}}$ represent, respectively, the initial and final lengths with temperature change $\Delta \mathrm{T}$, the CTE, $\alpha(\mathrm{T})$, is usually found to be nonlinear with temperature [17]. For example, silicon, which is widely used in MEMS structure, has the CTE found to be varied from $2.57 \mathrm{ppm} / \mathrm{K}$ at $293 \mathrm{~K}$ to $4.33 \mathrm{ppm} / \mathrm{K}$ at $1000 \mathrm{~K}[18]$.

In practice, MEMS silicon structure is usually glued onto a package carrier (e.g. polyimide, metals) using an adhesive material (e.g. super glue, M-bond). Furthermore, the whole strain gauge is then bonded onto a host infrastructure material (e.g. concrete, steel, aluminium, glass). Thus, the temperature effect on the capacitive sensor becomes extremely complex in 
TABLE I

COEFFICIENT OF THERMAL EXPANSION (CTE) OF SOME MATERIALS RELATED TO STRAIN SENSING

\begin{tabular}{lc}
\hline \hline \multicolumn{1}{c}{ Materials } & CTE $(\mathrm{ppm} / \mathrm{K})$ \\
\hline Polyimide & $\sim 20$ \\
Aluminium & $\sim 23$ \\
Super glue & $\sim 100$ \\
Steel & $\sim 11$ \\
Concrete & $\sim 12$ \\
Glass & $\sim 8.5$ \\
\hline \hline
\end{tabular}

strain sensing applications. The CTE of some of the abovementioned materials can be found in Table. I [17, 19]. The CTEs of these materials are a few times higher than that of silicon. Therefore, temperature effects will be exacerbated for these sensors, including the MEMS capacitive strain sensor.

Here, we introduce for the first time, an active compensation method based on a complement 2 -D capacitive structure design to help to reduce the thermal expansion issue in a MEMS capacitive sensor without any additional complex compensation circuit.

\section{DESIGN CONCEPT}

A concept of 2-D complement capacitive comb structure design is demonstrated in Fig. 2. The structures AB1 and AB2 are designed identically in term of distance, the number of interdigitated fingers, thickness, overlapping area and gap. The only difference is the fingers orientation layout, as clearly seen in Fig. 2. When the whole structure is heated, the substrate is expanding in all directions [20]. By neglecting the fringing effect, the capacitance between $\mathrm{AB} 1$ and $\mathrm{AB} 2, C_{\mathrm{AB} 1}, C_{\mathrm{AB} 2}$ can be found as:

$$
\begin{aligned}
& C_{A B 1}=\frac{2 N \varepsilon_{r} h(x-\Delta x 1)}{g} \\
& C_{A B 2}=\frac{2 N \varepsilon_{r} h(x+\Delta x 2)}{g}
\end{aligned}
$$

where $N$ is the number of interdigitated fingers on each branch, $h$ is the thickness of the fingers, $x$ is the initial overlapping length between fingers, $g$ is the gap between two adjacent fingers, and $\Delta x 1, \Delta x 2$ are the changes in the overlap between fingers due to substrate expansion under heating. It is noted

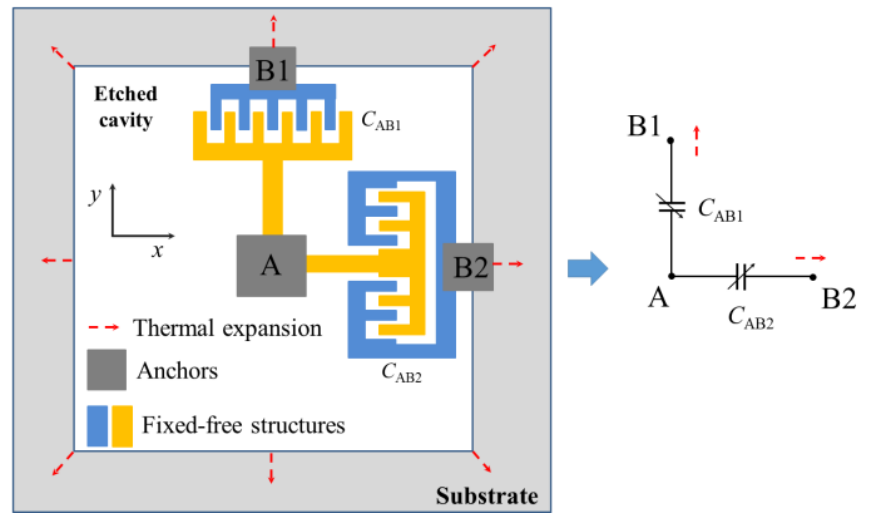

Fig. 2. An illustration of a temperature compensated capacitive sensor based on complement 2-D capacitive comb structure design.

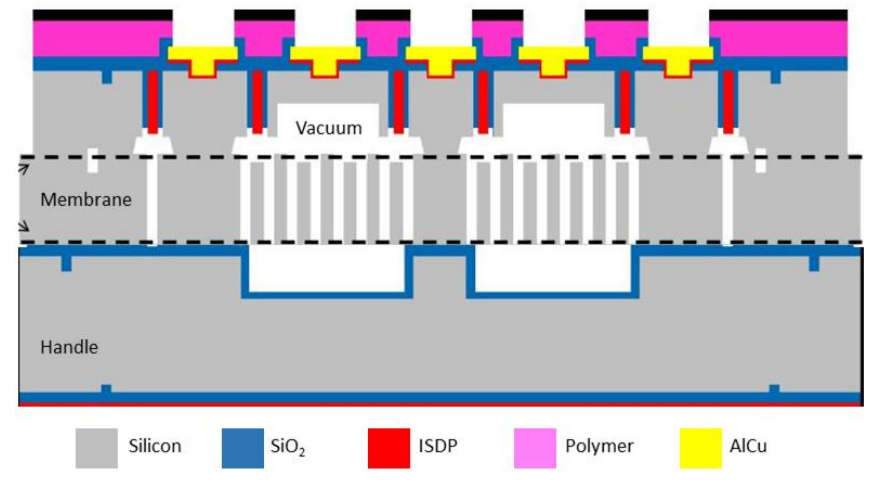

Fig. 3. MIDIS ${ }^{\mathrm{TM}}$ MEMS platform process by CMC.

that, in a comb structure, the fringing field effect is independent of the overlap area. The substrate contraction during cooling is following exactly similar behavior.

If the system is isotropic, expansion in $x$ and $y$ directions should be equal and hence, $\Delta x 1=\Delta x 2$. When B1 and B2 are connected, the capacitance factor caused by thermal expansion on two branches can be cancelled out and thus the total capacitance is independent of temperature variation to first order.

$$
C_{T}=C_{A B 1}+C_{A B 2}=\frac{4 N \varepsilon_{r} h(x)}{g}
$$

\section{THE SENSOR}

Based on the concept discussed in the previous section, MEMS capacitive sensors have been developed and fabricated on the MIDISTM Platform, which is being offered as MultiProject-Wafer (MPW) service through CMC Microsystems [21]. The process involves a $1.5 \mu \mathrm{m}$ minimum device feature size for a $30 \mu \mathrm{m}$ silicon device thickness incorporating waferlevel vacuum packaging. A process cross-section is provided in Fig. 3. Fig. 4 shows the SEM picture of the fabricated Design 1 before it was encapsulated. The design follows the thermal expansion compensation concept, as discussed above. Branches $\mathrm{AB} 3$ and $\mathrm{AB} 4$ are similar to $\mathrm{AB} 1$ and $\mathrm{AB} 2$, respectively. $\mathrm{B} 1$, B2, B3, B4 are connected by a top metal layer after encapsulation. In order to achieve a high-performance sensor

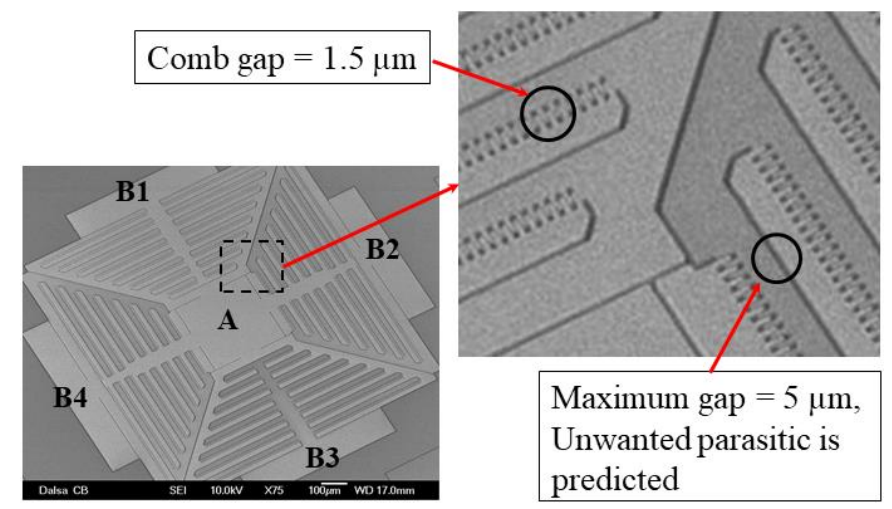

Fig. 4. SEM picture of the developed MEMS capacitive sensor with temperature compensation technique, Design 1. 


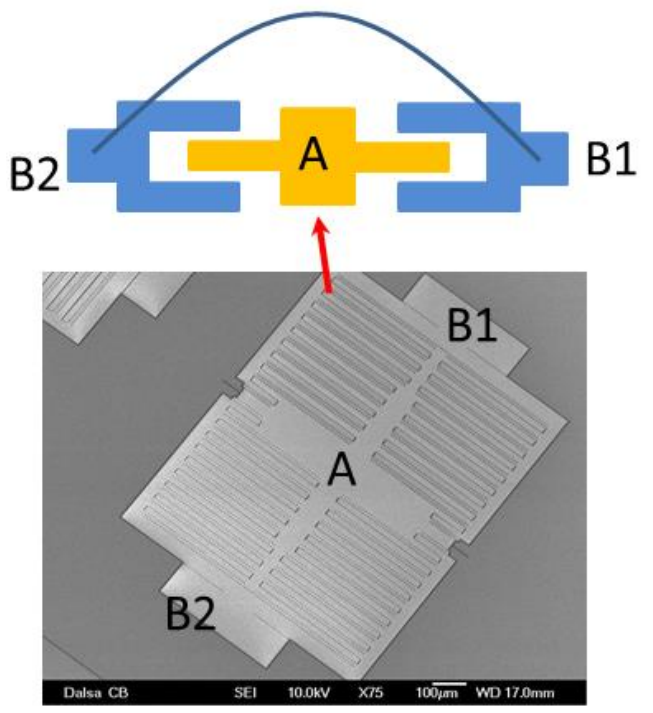

Fig. 5. SEM picture of the developed MEMS capacitive sensor without temperature compensation technique, Design 2.

TABLE II

DIMENSIONS AND ESTIMATED PARAMETERS OF THE MEMS CAPACITIVE SENSORS

\begin{tabular}{lll}
\hline \hline \multicolumn{1}{c}{ Description } & \multicolumn{1}{c}{ Design 1 } & \multicolumn{1}{c}{ Design 2 } \\
\hline Chip size & $1250 \times 1250 \mu \mathrm{m}^{2}$ & $1150 \times 820 \mu \mathrm{m}^{2}$ \\
Device thickness, $h$ & $30 \mu \mathrm{m}$ & $30 \mu \mathrm{m}$ \\
Total number of fingers, $N_{\mathrm{T}}$ & 2720 & 1428 \\
Capacitor finger gap, $g$ & $1.5 \mu \mathrm{m}$ & $1.5 \mu \mathrm{m}$ \\
Finger overlap, $x$ & $7.5 \mu \mathrm{m}$ & $10 \mu \mathrm{m}$ \\
Estimated comb capacitance & $7.22 \mathrm{pF}$ & $5.05 \mathrm{pF}$ \\
Estimated parallel parasitic & $1.8 \mathrm{pF}$ & $1.3 \mathrm{pF}$ \\
\hline \hline
\end{tabular}

with high capacitance and sensitivity, the interdigitated comb fingers are designed in a compact form, as shown in the zoomed-in sub-figure. The gap between fingers is designed at the process minimum of $g=1.5 \mu \mathrm{m}$. To reduce the parallel plate parasitic, the maximum gap is advised to be as large as possible. However, the maximum distance between adjacent structures of this process is limited at $5 \mu \mathrm{m}$, hence some unwanted high parasitic capacitance is predicted in this design. This will affect the sensitivity and resolution of the sensors. However, the 2-D complement capacitive concept described above is still applicable to the parallel plate capacitor. Therefore, the parasitic variations will also be cancelled out under this design.

In order to evaluate the effectiveness of the concept, a normal design without temperature compensation technique has also been developed to compare and contrast with the one in Fig. 4. The SEM picture is shown in Fig. 5. The general design structure concept of the interdigitated comb fingers layout is annotated on the top of the SEM figure. When temperature varies, the MEMS structure expands or contracts accordingly. Both $\mathrm{AB} 1$ and $\mathrm{AB} 2$ capacitance will either increase or decrease at the same time with temperature.

Table II shows designed dimensions and some estimated parameters of the two MEMS capacitive designs. The parallel plate parasitic is relatively high due to the limited maximum

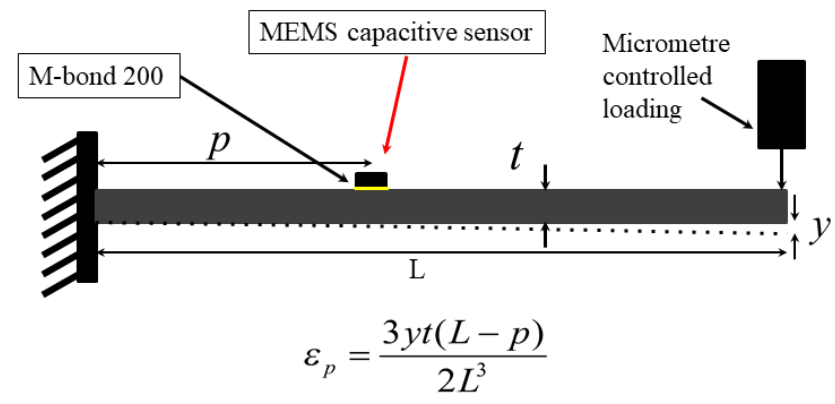

Fig. 6. Cantilever beam bending test setup for MEMS strain sensors.

gap in this process. It will eventually affect the sensitivity of the sensors as it travels in the opposite direction and inherits a nonlinear capacitance versus displacement relationship.

This is not an ideal comparison as Design 2 is not fullysymmetrical as Design 1. However, the total number of finger, $N_{\mathrm{T}}$, fingers gap, $g$, and the finger overlap, $x$, are mainly related to the range and resolution of the sensors. The temperature sensitivity is mainly related to the relative finger overlap, $\Delta x$. Hence, this relative comparison is still eligible to prove the concept.

\section{EXPERIMENTAL RESULTS AND DISCUSSION}

The fabricated sensors are attached by M-bond 200 on a cantilever beam bending test setup for strain sensing measurement, as shown in Fig. 6. Both sensors are on the same die. The die encapsulation is grounded to remove parasitic capacitance of the top metal layer, which is $\mathrm{AlCu}$. The chip is wire-bonded to a flexible PCB board for external connection. A cantilever beam with a length of $L$, and thickness of $t$ is anchored at one end with the MEMS sensors located at a position of $p$ from the anchor. The beam material is steel with length, $L$, of $220 \mathrm{~mm}$, thickness, $t$, of $3 \mathrm{~mm}$, and the sensors are located at $p=35 \mathrm{~mm}$. The displacement is measured by a micrometre, and the corresponding strain relationship can be found in the figure.

Fig. 7 presents the real-time measurement setup using a

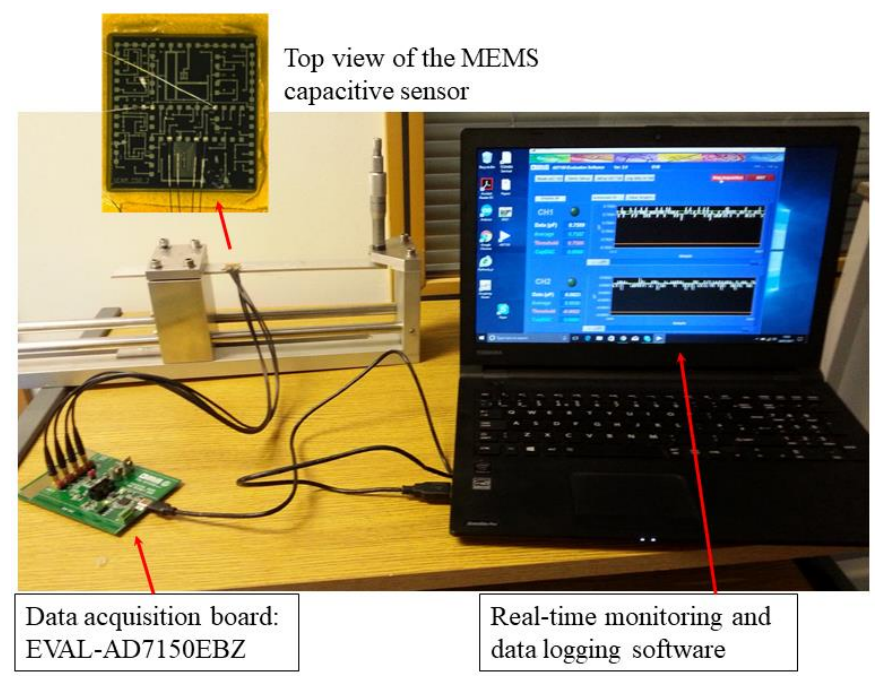

Fig. 7. Real-time monitoring and data logging strain sensing setup. 
TABLE III

EVAL-AD7150EBZ BOARD SETTING

\begin{tabular}{lcc}
\hline \hline \multicolumn{1}{c}{ Parameters } & Value & Unit \\
\hline Excitation frequency & 32 & $\mathrm{kHz}$ \\
$\begin{array}{l}\text { Conversion time (sampling } \\
\text { rate) }\end{array}$ & $5(200)$ & $\mathrm{ms} \mathrm{(Hz)}$ \\
Input range & 500 & $\mathrm{fF}$ \\
$\Delta-\Sigma$ CDC Resolution & 12 & $\mathrm{bit}$ \\
1 -bit resolution & 0.012 & $\mathrm{fF}$ \\
\hline \hline
\end{tabular}

commercial capacitive interface data acquisition board: EVALAD7150EBZ for capacitance-to-digital converter. It has two independent input channels. Hence two developed designs could be tested at the same time. Real-time graphic monitoring and data logging software is also provided.

The parameters for the EVAL-AD7150EBZ board is shown in Table III. The input range is set to the lowest possible range setting (500 fF). At this setting and with 12-bit CDC, the 1 bit resolution is $0.012 \mathrm{fF}$. The $\Sigma-\Delta$ modulator helps to significantly minimize the noise and therefore helps to enhance the measurement accuracy. The conversion time is set by default at 5 ms per sample.

The measured capacitance of the two designs are $10 \mathrm{pF}$ and $7.1 \mathrm{pF}$ for Design 1 and 2, respectively. They are $\sim 10 \%$ higher than the estimated values (including comb and parallel parasitic capacitance). The differences could be contributed by the wire bonds, PCB and coaxial cable connections parasitic.

Fig. 8 presents the measured output versus an applied input strain for both designs up to $800 \mu \varepsilon$ with a nonlinearity of less than $2 \%$ of full scale. Design 1 has a slightly higher sensitivity at $\sim 0.07 \mathrm{fF} / \mu \varepsilon$ than Design 2, which is measured at $0.05 \mathrm{fF} / \mu \varepsilon$. The equivalent Gauge Factors are found at 7 for both designs.

The temperature dependence characterization is carried out with an environmental chamber to evaluate the temperature compensation efficacy of the concept. Both designs are on the same die, which is attached to a steel bar with M-bond 200 glue. Fig. 9 shows the capacitance changes of the two designs for 10 samples each, with temperature varying from 25 to $130^{\circ} \mathrm{C}$. Error bars are around 0.03 to $0.05 \mathrm{fF}$. The CTC of Design 2

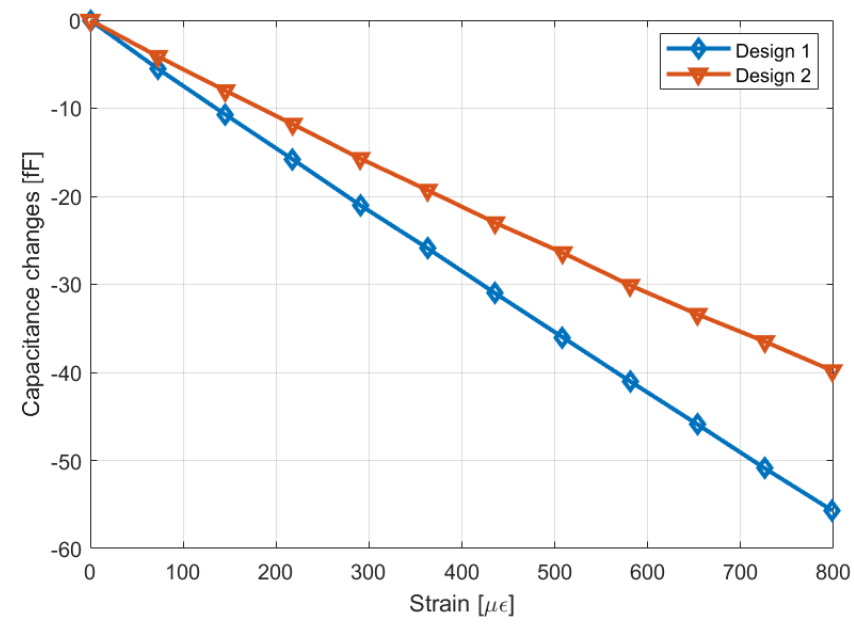

Fig. 8. Capacitance changes versus strain characterization of two developed MEMS sensors.

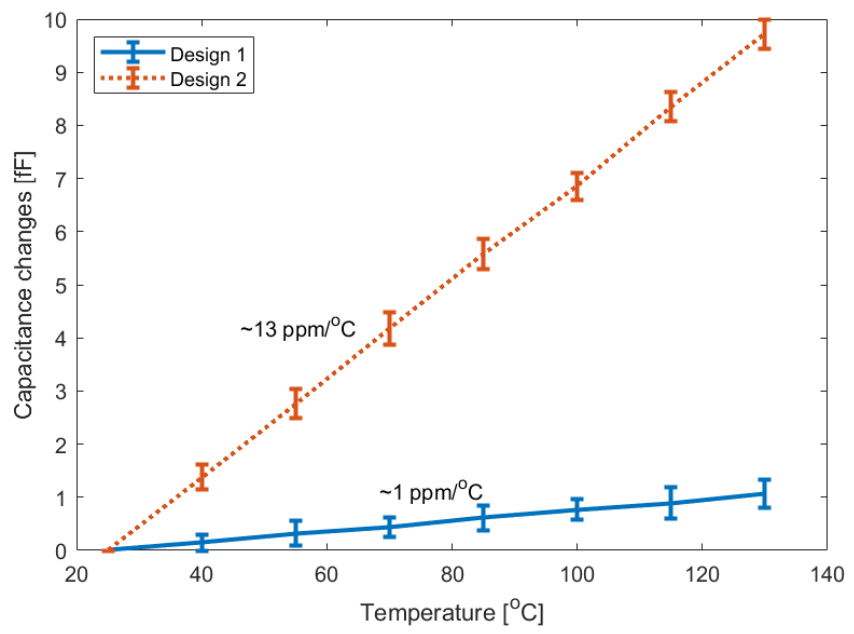

Fig. 9. Capacitance changes versus temperature.

(conventional design) is found at $\sim 13 \mathrm{ppm} /{ }^{\circ} \mathrm{C}$, which is similar to the CTE of steel of $\sim 11 \mathrm{ppm} /{ }^{\circ} \mathrm{C}$ as seen in Table. I. Whereas, the CTC of Design 1 (where the complement capacitive structure is utilized) is found at only $1 \mathrm{ppm} /{ }^{\circ} \mathrm{C}$. It is clearly shown the effectivity of the proposed method to reduce the temperature effect on MEMS capacitive strain sensor.

\section{CONCLUSION}

This paper presents the design concept and characterization results for a new method to compensate for the temperature effects of a capacitance-based strain gauge. The sensors were fabricated in a silicon MEMS foundry technology. The gauge factor is measured at 7 . The thermal effect has been assessed at a temperature up to $130^{\circ} \mathrm{C}$. The technique requires no additional circuit or special fabrication process.

The proposed method improves the CTC of the sensor by 13 times compared with the conventional design. Future work is required for the development of low-power front-end capacitive readout to improve resolution and sensitivity. Characterization of capacitive strain gauges for higher temperature operation (up to $1000^{\circ} \mathrm{C}$ ), long-term stability, and degradation mechanisms are also required in future studies.

This method is also applicable to other types of capacitive sensor, including proximity sensing, position sensing, humidity sensing, and tilt sensing. Further research on this topic on how to effectively employ this technique in those applications is being carried out.

If we were to design this study again, we would make Design 2 as symmetrical as Design 1 for a better comparison.

\section{REFERENCES}

[1] Ojuroye, Olivia O., et al. "Embedded capacitive proximity and touch sensing flexible circuit system for electronic textile and wearable systems." IEEE Sensors Journal, vol. 19, no. 16, 2019.

[2] J. Wang, Jiaqi, Z. Zheng, J. Chan, and J.T. Yeow, "Capacitive micromachined ultrasound transducers for intravascular ultrasound imaging." Microsystems \& Nanoengineering, vol. 6, no. 1, pp. 1-13, 2020.

[3] Anzinger, Sebastian, et al. "Low Power Capacitive Ultrasonic Transceiver Array for Airborne Object Detection." in IEEE 33rd 
International Conference on Micro Electro Mechanical Systems (MEMS), 2020.

[4] T. Emadi, and A.B. Douglas, "A novel $6 \times 6$ element MEMS capacitive ultrasonic transducer with multiple moving membranes for high performance imaging applications." Sensors and Actuators A: Physical, vol. 222, pp. 309-313, 2015.

[5] R.N. Miles, W. Cui, Q.T. Su, and D. Homentcovschi, "A MEMS lownoise sound pressure gradient microphone with capacitive sensing", Journal of Microelectromechanical Systems, vol. 24, no. 1, pp. 241248, 2015.

[6] Dong, Xianshan, et al. "Research on temperature characteristic of parasitic capacitance in MEMS capacitive accelerometer." Sensors and Actuators A: Physical, vol. 285, pp. 581-587, 2019.

[7] E.E. Aktakka, and K. Najafi, "A six-axis micro platform for in situ calibration of MEMS inertial sensors", in Proc. IEEE MEMS 29th International Conference, 2016.

[8] Mutharpavalar, Ashaashvini, A. Y. Ahmed, and Nursyarizal Mohd Nor. "Design, Modeling and Simulation of MEMS Resonator for Humidity Sensor Application." in Prof. IEEE Sensors Applications Symposium (SAS), 2020.

[9] N. Lazarus, S.S. Bedair, C.C. Lo, and G.K. Fedder, "CMOS-MEMS capacitive humidity sensor", Journal of Microelectromechanical Systems, vol. 19, no. 1, pp. 183-191, 2010.

[10] Rao, Kang, et al. "A high-resolution area-change-based capacitive MEMS tilt sensor." Sensors and Actuators A: Physical, vol. 313, 2020.

[11] L. Zhao, and E.M. Yeatman, "Micro capacitive tilt sensor for human body movement detection", in Proc. Body Sensor Networks, pp. 195200, 2007.

[12] Shirhatti, Vijay, et al. "High-range noise immune supersensitive graphene-electrolyte capacitive strain sensor for biomedical applications." Nanotechnology, vol. 30, no. 47, 2019.

[13] M. Suster, J. Guo, N. Chaimanonart, W. Ko, and D. J. Young, "A HighPerformance MEMS Capacitive Strain Sensing System", Journal of Microelectromechanical Systems, vol. 15, no. 5, Oct. 2006.

[14] J. Li, L.P. Jon, T. Szymon, G. Andrew, and S. Sanjay, "Interdigital capacitive strain gauges fabricated by direct-write thermal spray and ultrafast laser micromachining." Sensors and Actuators A: Physical, vol. 133, no. 1, pp. 1-8, 2007.

[15] Yaghootkar, B., Azimi, S., \& Bahreyni, B. (2017). A high-performance piezoelectric vibration sensor. IEEE Sensors Journal, vol. 17, no. 13, pp. 4005-4012, 2017.

[16] C.D. Do, A. Erbes, J. Yan, K. Soga, and A.A. Seshia, "Vacuum packaged low-power resonant MEMS strain sensor.", Journal of Microelectromechanical Systems, vol. 25, no. 5, pp. 851-858, 2016.

[17] F. Cverna, "ASM Ready Reference: Thermal properties of metals", ASM International, 2002.

[18] H. Watanabe, N. Yamada, and M. Okaji, "Linear Thermal Expansion Coefficient of Silicon from 293 to 1000 K", International Journal of Thermophysics, vol. 25, No. 1, Jan. 2004.

[19] G. Murray, "Handbook of Materials Selection for Engineering Applications", CRC Press, 1997.

[20] Watanabe, H., Yamada, N., \& Okaji, M. (2004). "Linear thermal expansion coefficient of silicon from 293 to $1000 \mathrm{~K}$ ", International journal of thermophysics, vol. 25, no. 1, pp. 221-236, 2014.

[21] https://www.cmc.ca/en/WhatWeOffer/Products/CMC-0020002990.aspx.

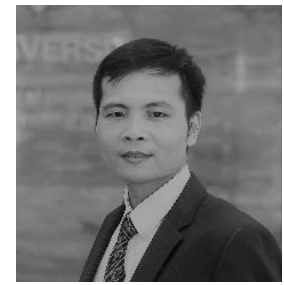

Cuong Do received the B. B.Sc. degree in electronics and telecommunication from Vietnam National University, Hanoi, in 2004, the M.Eng. degree in electronics from Chungbuk National University, South Korea, in 2007, and the Ph.D. degree in electronics from the Cork Institute of Technology, Ireland, in 2012.

From 2013 to 2017, he was a Research Associate with the University of Cambridge. Since 2019, he has been an Assistant Professor with the College of Engineering and Computer Science, VinUniversity, Hanoi. His research interests include fields of sensors and sensors fusion for healthcare applications.

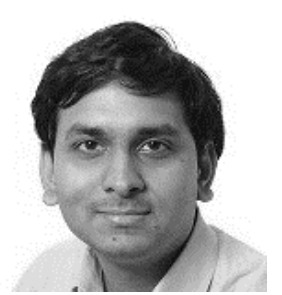

Ashwin A. Seshia received his BTech in Engineering Physics in 1996 from IIT Bombay, $\mathrm{MS}$ and $\mathrm{PhD}$ degrees in Electrical Engineering and Computer Sciences from the University of California, Berkeley in 1999 and 2002 respectively, and the MA from the University of Cambridge in 2008. He joined the faculty of the Engineering Department at the University of Cambridge in October 2002 where he is presently a Professor of Microsystems Technology and a Fellow of Queens' College. He is a Fellow of the Institute of Physics, a Fellow of the Institution for Engineering and Technology and a Fellow of the Institute of Electrical and Electronics Engineers. Prof Seshia currently serves on the editorial boards of the IEEE Journal of Microelectromechanical Systems and the IEEE Transactions on Ultrasonics, Ferroelectrics and Frequency Control, and the Executive Committee of the European Frequency and Time Forum. He received the 2018 IEEE Sensors Technical Achievement Award (Advanced Career - Sensor Systems) "for pioneering contributions to resonant microsystems with application to sub-surface density contrast imaging and energy harvesting systems". 